This item was submitted to Loughborough's Research Repository by the author.

Items in Figshare are protected by copyright, with all rights reserved, unless otherwise indicated.

\title{
Analysis of optimal operation of a fed-batch emulsion copolymerization reactor used for production of particles with core-shell morphology
}

\section{PLEASE CITE THE PUBLISHED VERSION}

http://dx.doi.org/10.1016/j.compchemeng.2014.02.021

\section{PUBLISHER}

(C) Elsevier

\section{VERSION}

AM (Accepted Manuscript)

\section{PUBLISHER STATEMENT}

This work is made available according to the conditions of the Creative Commons Attribution-NonCommercialNoDerivatives 4.0 International (CC BY-NC-ND 4.0) licence. Full details of this licence are available at: https://creativecommons.org/licenses/by-nc-nd/4.0/

\section{LICENCE}

CC BY-NC-ND 4.0

\section{REPOSITORY RECORD}

Paulen, Radoslav, Brahim Benyahia, M. Abderrazak Latifi, and Miroslav Fikar. 2015. "Analysis of Optimal Operation of a Fed-batch Emulsion Copolymerization Reactor Used for Production of Particles with Core-shell Morphology". figshare. https://hdl.handle.net/2134/17590. 


\title{
Analysis of optimal operation of a fed-batch emulsion copolymerization reactor used for production of particles with core-shell morphology
}

\author{
R. Paulen ${ }^{\mathrm{a}, \mathrm{b}, \mathrm{c}}$, B. Benyahia ${ }^{\mathrm{d}}$, M.A. Latifi ${ }^{\mathrm{a}}$, M. Fikar $^{\mathrm{b}}$ \\ ${ }^{a}$ Laboratoire Réactions et Génie des Procédés CNRS - ENSIC, Université de Lorraine, \\ UPR 6811 CNRS, 1 rue Grandville, Nancy, France \\ ${ }^{b}$ Faculty of Chemical and Food Technology, Slovak University of Technology in \\ Bratislava, Radlinskeho 9, Bratislava, Slovakia \\ ${ }^{c}$ Department of Chemical and Biochemical Engineering, Technische Universität \\ Dortmund, Emil-Figge-Strasse 70, 44221 Dortmund, Germany \\ ${ }^{d}$ Department of Chemical Engineering, Loughborough University, Loughborough, \\ Leicestershire LE11 3TU, UK
}

\begin{abstract}
In this paper dynamic optimization of a lab-scale semi-batch emulsion copolymerization reactor for styrene and butyl acrylate in the presence of a chain transfer agent (CTA) is studied. The mathematical model of the process, previously developed and experimentally validated, is used to predict the glass transition temperature of produced polymer, the number and weight average molecular weights, the monomers global conversion, the particle size distribution, and the amount of residual monomers. The model is implemented within gPROMS environment for modeling and optimization. It is desired to compute feed rate profiles of pre-emulsioned monomers, inhibitor and CTA that will allow the production of polymer particles with prescribed core-shell morphology with high productivity. The results obtained for different oper-
\end{abstract}

\footnotetext{
*Corresponding author. Tel.: +421 259325 730; fax: +421 259325340.

Email address: radoslav.paulen@stuba.sk (R. Paulen) 
ating conditions and various additional product specifications are presented. The resulting feeding profiles are analyzed from the perspective of the nature of emulsion polymerization process and some interesting conclusions are drawn.

Keywords: Emulsion Copolymerization, Dynamic Optimization, Chain Transfer Agent, Fedbatch Process, Control Vector Parameterization

\section{Introduction}

Emulsion (co)polymerization represents an important class of industrial processes used to produce latexes of multiple uses (e.g. paints, adhesives, coatings, etc.) [1]. This technique has several advantages over other types of polymerization (e.g. bulk and solution polymerization) and continues to attract an increasing interest for both industrial and academic communities. Emulsion polymerization involves a reaction driven by radical kinetics. A typical emulsion polymerization formulation comprises dispersion medium, which typically consists of water, monomer(s), initiator, and surfactant. The advantages of emulsion polymerization result mostly from the multiphase and compartmentalized nature of the process which allows the production of polymers with high molecular weights at high polymerization rates with

high quality products. Another key advantage of employing emulsion type of polymerization process is a very good controllability of the temperature. This feature is ensured by the excellent heat conducting properties of the large amount of water present in the reactor.

However, emulsion polymerization reactor represents a rather complex system from modeling, simulation, and optimization point of view. This 
complexity arises from the presence of non-linear and discontinuous behavior rending the development of optimization procedures of emulsion polymerization reactions a very challenging task. There is a rich literature on modeling of emulsion polymerization processes, starting with the conventional Smith-Ewart model [2], which identifies three stages in emulsion polymerization process (nucleation, particles growth, and end of polymerization). The later models developed for emulsion polymerization have different degrees of complexity [3, 4], depending upon their scope and application. The most representative ones are reviewed by Gao and Penlidis [5] and Chern [6]. In this study, we use the mathematical model developed in our recent research activities [7]. The model is of hybrid type with several differential and algebraic equations and with state-dependent switching being present. From a numerical point of view, it possesses rather a stiff behavior, i.e. due to the presence of discontinuities and algebraic equations. On the other hand, it has proven to be a relatively accurate representation of the experimental behavior of the reactor since the most important product properties were shown to be predicted reliably.

Molecular weight distribution (MWD), glass transition temperature $\left(T_{g}\right)$, particle size distribution (PSD), and particle morphology are the main parameters which strongly govern the end-use properties of the products (latex and polymer). For example, PSD is strongly correlated to the rheological properties, adhesion and film-forming properties of the final products. On the other hand, MWD affects important end-use properties of the film, such as elasticity, strength, toughness, and solvent resistance.

One of the most challenging problems of the operation of emulsion poly- 
merization reactors is the development of an optimization and control strategy that ensures the production of nanoparticles with the targeted end-use properties, particularly at the industrial scale. In this study, we are concerned with the problem of controlling the particle morphology evolution (during the copolymerization reaction) such that the core-shell morphology of produced particles is established through the tracking of a desired glass transition temperature profile of produced polymers. This tracking is carried out by feeding control of monomers, inhibitor, and CTA under maintained isothermal conditions. Typical industrial applications require polymers to be produced under starving conditions which obviously limit the feeding of the monomer and thus they create an implicit restrictions on the quality of produced polymers. We may subsequently conclude that the control of morphological properties of the polymer particles represents an interesting yet not fully solved problem of process control similar to control problems recognized e.g. in automatically controlled crystallizations. During the last few years, our research group has developed novel optimization and control strategies [8] to tailor the latex properties such as morphology and gel transition temperatures at the nanoscale, particularly for a specific need in the paint industry. Some of these strategies have been successfully applied experimentally. More novel strategies are presented in the present study and they are compared to our previous works to show a real potential for further improvement of the ability to tailor the latex particles at the nano-scale, while maintaining high yield of the polymerization. As such, this paper is anticipated to reach not only the community working on all types of emulsion polymerization but a wider community of researchers and industrialists 
interested in nano-synthesis.

Beside pure experimental studies [9], various properties of copolymerization reactive systems were analyzed with respect to the particle morphology control $[10,11,12]$. In our previous study [8], we formulated the problem of optimal operation of copolymerization system as a multi-objective problem since two conflicting objectives, i.e. achieving required quality and maximal amount of the produced polymer, were involved. In the present paper, we aim to provide an analysis of optimal feeding policy of a polymerization reactor for control of end-use properties of emulsion copolymerization products. Our analysis is based on dynamic optimization of a tendency model for the emulsion copolymerization of styrene and butyl acrylate in the presence of $n$-dodecyl mercaptan as chain transfer agent. The model predicts the global monomers conversion, the number and weight average molecular weights, the average diameter of polymer particles, and the residual amounts of monomers.

This paper is an extended version of the contribution [13] presented at the $23^{\text {rd }}$ European Symposium on Computer Aided Process Engineering (ESCAPE). It is organized as follows. In the next section, the process model is described in compact form where only the crucial parts for the analysis are presented. Next, process optimization problem is stated and solved. An analysis of the optimal solution is performed for various scenarios of operational requirements. Finally, the results are discussed and analyzed. 


\section{Process model}

The copolymerization reaction takes place in a reactor equipped with a cooling jacket which helps to maintain isothermal conditions in the system. The reaction is carried out in fed-batch mode where the feeding stream consists of pre-emulsioned mixture of monomers (styrene and butyl acrylate), inhibitor, and CTA. An initiator is fed to the reactor by means of a separate inlet stream. The mathematical model of the considered emulsion copolymerization reaction was derived, identified and validated in one of our previous studies [7]. This section presents the model in compact form where we show its crucial features, i.e. considered reaction mechanism and resulting model equations. The detailed model can be found in the original modeling paper [7].

Emulsion polymerization is driven by radical mechanisms where the monomers are mainly located in droplets dispersed in an aqueous phase. These droplets are stabilized by a surfactant. The initiator is soluble in the water phase which contains an excess of surfactant mainly in its micellar form. The initiator decomposes in the aqueous phase and generates primary radicals. In conventional emulsion polymerization monomers with very low water solubility are used and this leads to two types of nucleation. In the case of homogeneous nucleation, radicals propagate beyond their water solubility, they precipitate and are then stabilized by the available emulsifier present in the medium. On the other hand, when micellar nucleation takes place, primary radicals enter to the micelle and lead to polymer particles in which propagation, termination, inhibition and chain transfer reactions take place. The monomers needed for the reactions are provided by the droplets. 


\subsection{Kinetic scheme}

As mentioned above, emulsion polymerization involves mostly radical reactions being present in both aqueous and organic phases.

\subsubsection{Aqueous phase}

The reactions that take place in the aqueous phase are the following :

$\begin{array}{rrll}\text { Initiation } & I_{2} & \stackrel{k_{d}}{\rightarrow} & 2 R_{a q}^{\bullet} \\ \text { Inhibition } & R_{a q}^{\bullet}+Z_{a q} & \stackrel{k_{Z_{a q}}}{\rightarrow} & P+Z_{a q}^{\bullet} \\ \text { Nucleation } & R_{a q}^{\bullet}+\text { micelle } & \stackrel{k_{N}}{\rightarrow} & \text { particle }+R^{\bullet} \\ \text { Radical absorption } & R_{a q}^{\bullet} & \stackrel{k_{c p}}{\rightarrow} & R^{\bullet}\end{array}$

\subsubsection{Organic phase (particles)}

The following reactions take place in the organic phase :

$$
\text { Propagation } \quad R_{i}^{\bullet}+M_{j} \stackrel{k_{p i j}}{\rightarrow} R_{j}^{\bullet}
$$

Termination by combination $\quad R_{i}^{\bullet}+R_{j}^{\bullet} \stackrel{k_{t c i j}}{\rightarrow} P$

Termination by disproportionation $\quad R_{i}^{\bullet}+R_{j}^{\bullet} \stackrel{k_{t d i j}}{\rightarrow} 2 P$

Inhibition $\quad R_{i}^{\bullet}+Z_{p} \stackrel{k_{Z p}}{\rightarrow} P+Z_{p}^{\bullet}$

Transfer to monomers $\quad R_{i}^{\bullet}+M_{j} \stackrel{k_{\text {trmij }}}{\rightarrow} P+R_{j}^{\bullet}$

Transfer to transfer agent $R_{i}^{\bullet}+C T A_{p} \stackrel{k_{C T A p}}{\rightarrow} P+C T A_{p}^{\bullet}$

$$
\text { Radical desorption } \quad R^{\bullet} \stackrel{k_{d e s}}{\rightarrow} R_{a q}^{\bullet}
$$

\subsection{Mass balance equations}

The mass balance is presented in a general form for a semi-batch process using the reaction rates mentioned above. These equations could be easily 
simplified for the case of a batch process.

$$
\begin{aligned}
\frac{\mathrm{d} V_{R}}{\mathrm{~d} t} & =Q_{f}+Q_{I f}+\sum_{i=1,2}\left(\frac{1}{\rho_{p i}}-\frac{1}{\rho_{i}}\right) M_{M}^{i}\left(\mathcal{R}_{p i}+\mathcal{R}_{t r m i}\right), \\
\frac{\mathrm{d} M_{i}}{\mathrm{~d} t} & =-\mathcal{R}_{p i}-\mathcal{R}_{t r m i}+Q_{f}\left[M_{i}\right]_{f}, \quad \forall i=1,2 \\
\frac{\mathrm{d} M_{T i}}{\mathrm{~d} t} & =Q_{f}\left[M_{i}\right]_{f}, \quad \forall i=1,2 \\
\frac{\mathrm{d} I}{\mathrm{~d} t} & =-\mathcal{R}_{d}+Q_{I f}[I]_{f}, \\
\frac{\mathrm{d} Z}{\mathrm{~d} t} & =-\left(\mathcal{R}_{Z p 1}+\mathcal{R}_{Z p 2}\right)+Q_{f}[Z]_{f}, \\
\frac{\mathrm{d} C T A}{\mathrm{~d} t} & =-\mathcal{R}_{C T A p 1}-\mathcal{R}_{C T A p 2}+Q_{f}[C T A]_{f}, \\
\frac{\mathrm{d} S}{\mathrm{~d} t} & =Q_{f}[S]_{f}, \\
\frac{\mathrm{d} N_{p}}{\mathrm{~d} t} & =\mathcal{R}_{N}, \\
\frac{\mathrm{d} R_{p 1}}{\mathrm{~d} t} & =\left(\mathcal{R}_{N}+\mathcal{R}_{c p}\right) f_{a q 1}-\mathcal{R}_{p 12}+\mathcal{R}_{p 21}-\mathcal{R}_{t r m 12} \\
& +\mathcal{R}_{t r m 21}-\mathcal{R}_{Z p 1}-\mathcal{R}_{d e s 1}-\left(\mathcal{R}_{T 11}+\mathcal{R}_{T 12}\right), \\
\frac{\mathrm{d} R_{p 2}}{\mathrm{~d} t} & =\left(\mathcal{R}_{N}+\mathcal{R}_{c p}\right) f_{a q 2}-\mathcal{R}_{p 21}+\mathcal{R}_{p 12}-\mathcal{R}_{t r m 21} \\
& +\mathcal{R}_{t r m 12}-\mathcal{R}_{Z p 2}-\mathcal{R}_{d e s 2}-\left(\mathcal{R}_{T 22}+\mathcal{R}_{T 21}\right),
\end{aligned}
$$




\subsection{Molecular weight distribution}

In order to predict the molecular weight distribution, the classical moment equations are used and given as

$$
\begin{aligned}
\frac{\mathrm{d}\left(N_{p} \tilde{n}\right)}{\mathrm{d} t}= & 2 \mathcal{R}_{c p} \bar{n}-\left(\frac{2 \tilde{\tilde{n}}}{\tilde{n}}+1\right) \mathcal{R}_{T}-2 \frac{\tilde{n}}{\bar{n}}\left(\mathcal{R}_{Z p}+\mathcal{R}_{d e s}\right), \\
\frac{\mathrm{d}\left(N_{p} \bar{n}\right)}{\mathrm{d} t}= & \mathcal{R}_{N}+\mathcal{R}_{c p}-\left(\mathcal{R}_{Z p}+\mathcal{R}_{T}+\mathcal{R}_{d e s}\right) \\
\frac{\mathrm{d}\left(N_{p} \bar{n} \lambda_{1}\right)}{\mathrm{d} t}= & \mathcal{R}_{N}+\mathcal{R}_{c p}-\mathcal{R}_{d e s}+\mathcal{R}_{p}-\left(\mathcal{R}_{Z p}+\mathcal{R}_{T}\right) \lambda_{1} \\
+ & \left(\mathcal{R}_{t r m}+\mathcal{R}_{C T A p}\right)\left(1-\lambda_{1}\right) \\
+ & \left(\mathcal{R}_{t r m}+\mathcal{R}_{C T A p}\right)\left(1-\lambda_{2}\right)-\left(\mathcal{R}_{Z p}+\mathcal{R}_{T}\right) \lambda_{2} \\
\frac{\mathrm{d}\left(N_{p} \bar{n} \lambda_{2}\right)}{\mathrm{d} t}= & \mathcal{R}_{N}+\mathcal{R}_{c p}-\mathcal{R}_{d e s}+\mathcal{R}_{p}\left(1+2 \lambda_{1}\right) \\
\frac{\mathrm{d} N_{m}}{\mathrm{~d} t}= & \mathcal{R}_{Z p}+\mathcal{R}_{t r m}+\mathcal{R}_{T D}+\mathcal{R}_{C T A p}+\frac{\mathcal{R}_{T C}}{2} \\
\frac{\mathrm{d}\left(N_{m} L_{1}\right)}{\mathrm{d} t}= & \lambda_{1}\left(\mathcal{R}_{Z p}+\mathcal{R}_{t r m}+\mathcal{R}_{T D}+\mathcal{R}_{C T A p}+\mathcal{R}_{T C}\right) \\
\frac{\mathrm{d}\left(N_{m} L_{2}\right)}{\mathrm{d} t}= & \lambda_{2}\left(\mathcal{R}_{Z p}+\mathcal{R}_{t r m}+\mathcal{R}_{T D}+\mathcal{R}_{C T A p}\right) \\
& +\mathcal{R}_{T C}\left(\lambda_{2}+\lambda_{1}^{2}\right)
\end{aligned}
$$

The number and weight average molecular weights, $\bar{M}_{n}$ and $\bar{M}_{w}$, can be then easily computed using the following formulas

$$
\begin{aligned}
& \bar{M}_{n}=\bar{M} L_{1}, \\
& \bar{M}_{w}=\bar{M} \frac{L_{2}}{L_{1}},
\end{aligned}
$$

where $\bar{M}$ is the average molecular weight of the monomeric unit given by

$$
\bar{M}=\frac{\sum_{i=1,2}\left(M_{T i}-M_{i}-R_{a q} f_{a q i}\right) M_{M}^{i}}{\sum_{i=1,2}\left(M_{T i}-M_{i}-R_{a q} f_{a q i}\right)} .
$$




\subsection{Glass transition temperature}

The control of the product quality during emulsion polymerization requires the monitoring of a large set of parameters related to end-use properties. Some of these properties are often described through distributed characteristics such as MWD and/or the copolymer composition distribution. The latter is the case of the glass transition temperature, $T_{g}$, which depends strongly on the microstructure of the macromolecules, particularly the copolymers. To complete the model, the glass transition temperature is expressed according to Fox's equation [14].

$$
\left[T_{g}+\frac{a}{\bar{M}_{n}}\right]^{-1}=\sum_{i=1,2} \frac{W_{i}}{T_{g i}}
$$

where $a$ is an adjustment parameter (Fox and Flory constant), $T_{g i}$ is the glass transition temperature of the homopolymer of $i$ th monomer and $W_{i}$ stands for the mass fractions of $i$ th monomer in the copolymer defined by

$$
W_{i}=\frac{\left(M_{T i}-M_{i}-R_{a q} f_{a q i}\right) M_{M}^{i}}{\sum_{i=1,2}\left(M_{T i}-M_{i}-R_{a q} f_{a q i}\right) M_{M}^{i}},
$$

Another possibility is to use the so-called instantaneous glass transition temperature, $T_{g}^{\text {ins }}$, defined by

$$
\frac{1}{T_{g}^{\text {ins }}}=\sum_{i=1,2} \frac{W_{i}^{\text {ins }}}{T_{g i}}
$$

where $W_{i}^{\text {ins }}$ is the instantaneous mass fraction of the $i$ th monomer in the copolymer defined as

$$
W_{i}^{\mathrm{ins}}=\frac{R_{p i} M_{M}^{i}}{R_{p 2} M_{M}^{2}+R_{p 2} M_{M}^{2}} .
$$




\subsection{Global conversion and residual mass fraction}

The global mass (monomers) conversion is given by

$$
X=\frac{\sum_{i=1,2}\left(M_{T i}-M_{i}\right) M_{M}^{i}}{\sum_{i=1,2} M_{T i} M_{M}^{i}} .
$$

and the residual mass fraction of $i$ th monomer is defined as

$$
F_{i}=\frac{M_{i} M_{M}^{i}}{\sum_{i=1,2} M_{i} M_{M}^{i}}
$$

\subsection{Discussion}

The resulting model of copolymerization process involves 18 ordinary differential equations and several algebraic equations (surfactant and volume partition equations). Moreover, the model exhibits a hybrid (switching) nature. This is mainly due to the gel and glass effects as well as droplets disappearance process. Finally, we can state that the copolymerization process is modeled as a set of discontinuous differential-algebraic equations (DAEs). These properties make simulation and optimization of this process a very challenging task since both activities require numerical integration of stiff systems. To cope with the stiffness of the process model we normalized the state variables which is being found by using heuristics (mass balance states are scaled by division with their respective initial conditions) and trial-anderror approach for the rest of dynamical variables.

\section{Goal of process operation}

The goal of this study is to investigate time-dependent feed flowrate (of pre-emulsion of monomers, inhibitor, CTA, and surfactant) of the fed-batch 
copolymerization reactor. It is desired to produce a latex particles with coreshell morphology and prescribed quality (represented by glass transition temperature of core and shell) together with maximizing the overall conversion of monomers at final time which maximizes the amount of produced copolymer and simultaneously minimizes the residual monomer content in the particles.

The optimization goal is schematically illustrated in Fig. 1 where $Q_{f}$ stands for the feed rate of pre-emulsion (monomers, inhibitor, CTA, and surfactant) to the reactor. The first stage of the process is characterized by the formation of seeds (primary copolymer particles) and it runs in batch mode. This stage terminates (at time $t_{0}$ ) once the monomers overall conversion reaches $90 \%$.

In the second step, it is desired to form a core of the latex particles such that the glass transition temperature is as close as possible to $T_{g 1}=276.15 \mathrm{~K}$ (solid line in the middle plot). We consider the reaction to be in fed-batch mode with time-dependent feed rate of pre-emulsioned monomers, inhibitor, and CTA. We impose a path constraint on the monomers overall conversion in a way it can vary only in a close neighborhood of $90 \%$ (sketched by solid lines and arrows in lower plot). This represents a requirement for starving conditions, which allows better control of the copolymerization reactions within the particles. The closeness of this neighborhood can be loosened or strengthened by adjusting the value of the integral of deviation from starving conditions (here parameter $\varepsilon>0$ ).

The use of the relaxation for starving condition constraint is motivated by a simple observation when Eqs. (6) and (10) are compared. It can be seen that these key qualitative and quantitative indicators of produced copolymer 

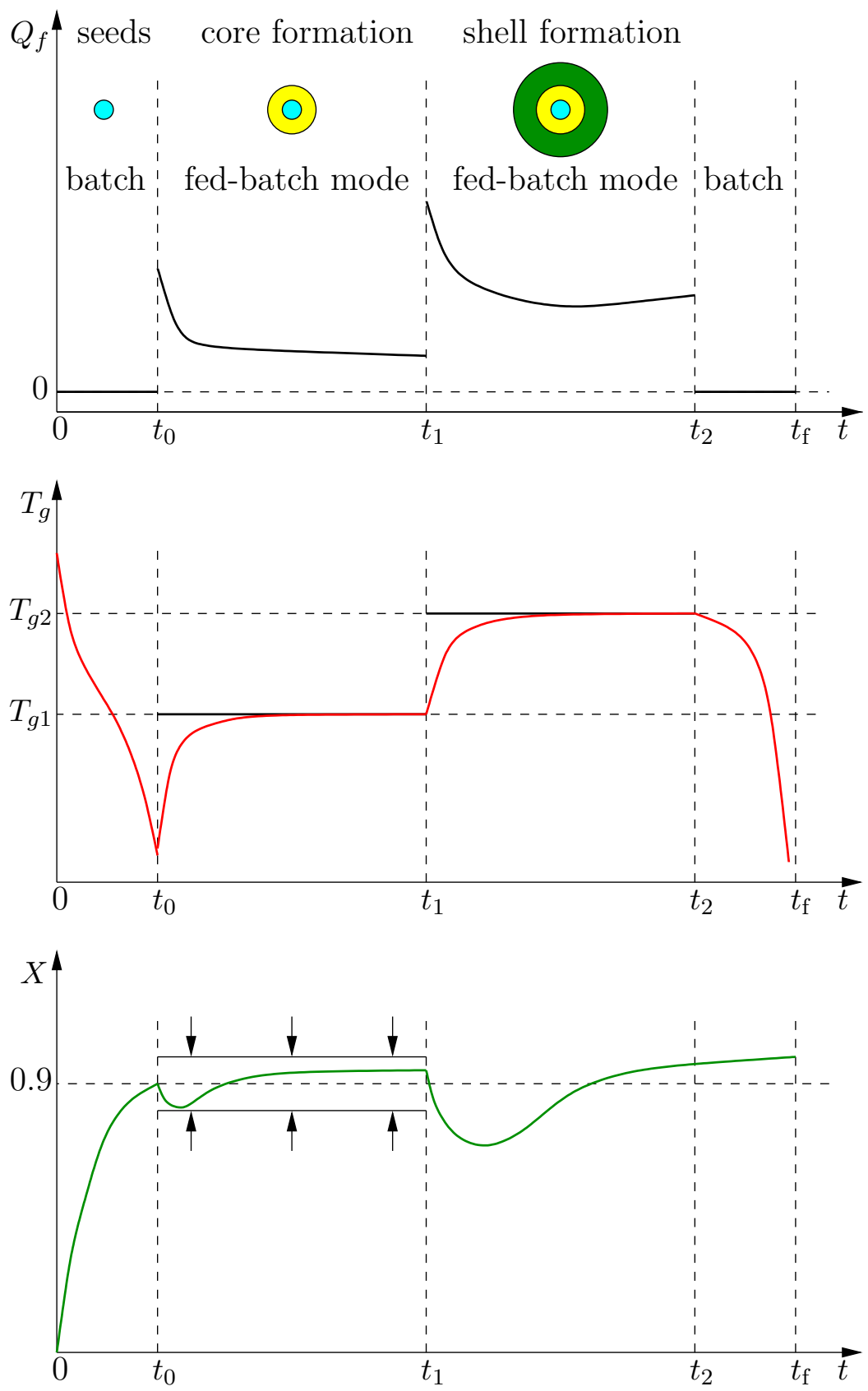

Figure 1: Expected profiles of the feed over various process phases (upper plot), glass transition temperature (middle plot), and monomers overall conversion (lower plot). 
need to adjust the same variables, i.e. total and instantaneous amounts of monomer (here it can be assumed, without the loss of generality, that the molar fraction of monomer in aqueous phase is negligible). After some rearrangement of the aforementioned equations, we can observe that the (instantaneous) glass transition temperature and (instantaneous) global monomers conversion are coupled.

The only degrees of freedom of relation between $T_{g}$ and $X$, that can be influenced by feeding rate, are concentrations of monomers since the reaction is maintained isothermal. The dynamic evolution of monomers concentrations is, at the same time, constrained by reaction conditions (e.g. rate of polymerization of a particular monomer). Moreover, it is assumed that the monomers are added with pre-designed constant ratio. We can then conclude that the satisfactory tracking of a $T_{g}$ profile would be possible only if the starving condition constraint is relaxed (back-off). This type of trade-off is a typical outcome of the similar analyses in chemical production systems (e.g. conversion vs. selectivity trade-offs).

The second step is ended (at time $t_{1}$ ) once the (average) diameter of the produced particles $d_{p}$ is equal to a desired value $d_{p 1}$ or when the half of the allowed feed amount is consumed (the volume in the reactor is then fixed at this stage to prescribed value $V_{R 1}$ ). The former condition applies in the cases when core-shell morphology is specifically connected to the diameter of produced particles while the latter one is imposed when the restriction of upper volume limit in the reactor is present. We will investigate the effects of both conditions in this study.

At the shell formation stage, the desired transition temperature is raised 
to $T_{g 2}=283.15 \mathrm{~K}$ (solid line in the middle plot) and we do not impose any path constraint on state variables. This condition is taken into account in order to analyze the same control requirement as in second step without any restrictions in the form of path constraints. The third step ends once the diameter of the produced particles is equal to a desired value $d_{p 2}$ or when the upper limit on the volume in the reactor $V_{R, \max }$ is reached. The final step takes place in the batch mode and the goal is to maximize the monomers overall conversion. At the final time, we additionally impose constraints on number and weight average molecular weights which are the indicators of polymer quality as already mentioned.

Mathematically, this problem may be formulated as

$$
\min _{Q_{f}(t)} \int_{t_{0}}^{t_{1}}\left[T_{g}(t)-T_{g 1}\right]^{2} \mathrm{~d} t+\int_{t_{1}}^{t_{2}}\left[T_{g}(t)-T_{g 2}\right]^{2} \mathrm{~d} t-\int_{t_{2}}^{t_{\mathrm{f}}} \omega \dot{X}(t) \mathrm{d} t,
$$

s.t. process model (Eqs. (1) and (2)),

initial conditions,

$$
\begin{aligned}
& X\left(t_{0}\right)=0.9 \\
& \int_{t_{0}}^{t_{1}}[X(t)-0.9]^{2} \mathrm{~d} t \leq \varepsilon \\
& d_{p}\left(t_{1}\right)=d_{p 1}, \quad \text { or } \quad V_{R}\left(t_{1}\right)=V_{R 1}, \\
& d_{p}\left(t_{2}\right)=d_{p 2}, \quad \text { or } \quad V_{R}\left(t_{2}\right)=V_{R, \max }, \\
& M_{n}\left(t_{\mathrm{f}}\right) \leq 4 \times 10^{4}, \\
& M_{w}\left(t_{\mathrm{f}}\right) \leq 1.5 \times 10^{5}, \\
& 0 \leq Q_{f}(t) \leq Q_{f, \max } \equiv 8.33 \times 10^{-7} \mathrm{~m}^{3} \mathrm{~s}^{-1},
\end{aligned}
$$

where $V_{R}$ represents a total volume engaged in the reactor, $d_{p}$ stands for average diameter of produced polymer particles and $\bar{M}_{n}$ and $\bar{M}_{w}$ are the number 
and weight average molecular weights of the produced polymer respectively. Here $\omega$ is a weighting factor of the last term in the objective function. It is set equal to 1000 in order to balance the difference in magnitude between the first two terms in the objective function and the last one. The last set of inequalities expresses operational conditions of the real plant which was used for validation of the process model and for experimental control of the process in our previous studies $[7,8]$. Here $Q_{f, \max }$ denotes the maximal flowrate achievable with the present plant instrumentation.

\subsection{Dynamic optimization}

There are two main classes of deterministic dynamic optimization approaches, analytical and numerical methods, which can be used in order solve optimization problem (12). The strength of analytical methods lies in finding a solution to dynamic optimization problem in its original infinitedimensional form. These methods are, however, rather impractical to use for highly non-linear and higher order problems. On the other hand, dynamic optimization of complex processes like emulsion copolymerization processes, may be carried out in much more convenient (and, in fact, only admissible and computationally tractable) way by using numerical techniques. These techniques consist in the transformation of the original problem of optimal control into a finite-dimensional non-linear programming problem (NLP) which is then solved by means of a gradient-based NLP solver.

We use a direct numerical optimization approach, i.e. control vector parameterization method (CVP) [15], to solve the defined dynamic optimization problem. This method transforms formerly infinite-dimensional dynamic optimization problem into a finite-dimensional form of NLP problem by apply- 
ing a piece-wise polynomial approximation of the control variables. We apply a piece-wise constant (PWC) discretization of the control profile $Q_{f}(t)$. The control profile is divided into finite number of PWC segments whose values (constant control over the segment) and lengths (time duration) are subject to optimization. We restrict the time duration of each segment to be at least 30 seconds which is a value technologically admissible with regard to the construction of the real-world system (capacity of the pumps).

Employing the CVP method, the solution of the optimization problem (12) can be found by means of combined numerical simulation and optimization tools. There are many packages available that provide an efficient implementation of various algorithms. These include the state-of-the-art packages such as gPROMS [16], ACADO [17], Jacobian, PROPT [18], MUSCOD-II [19], or the tools developed in our group, such as DYNO [20] or DYNOPT [21]. The gPROMS environment for simulation and optimization offers an intuitive way to simulate mathematical models and it proved to be very efficient for optimization of hybrid and large-scale systems. Its features include solving systems of DAEs, automatic root finding of switching functions, when the process model is of discontinuous nature, as well as automatic sensitivity functions generation and evaluation which can be exploited for efficient run and implementation of CVP method [22]. As a consequence, gPROMS has gained a lot of interest from both academia and industry over the last decade. This is a key feature because of which gPROMS has been selected for performing the dynamic optimization in our study in order to reach a larger community of scientist and engineers. However, similar results could be obtained using any other standard dynamic optimization package such as 
Table 1: Composition of the initial load and the feed stream of the reactor.

\begin{tabular}{lcc} 
Component & Initial load $[\mathrm{g}]$ & Feed $[\mathrm{g}]$ \\
\hline Water & 171 & 399 \\
Butyl acrylate & 12 & 48 \\
Styrene & 12 & 48 \\
Initiator & 1 & 0 \\
Emulsifier & 3 & 12 \\
CTA & 0.12 & 0.48 \\
\hline
\end{tabular}

the aforementioned ones.

\section{Results and discussion}

Table 1 presents the composition of the initial load and pre-emulsioned feed stream which we use in our calculations. We investigate the optimal feeding profiles for several scenarios which arise from the nature of the process as well as from the optimization problem definition. These are summarized in Table 2.

The first scenario (S1) investigates the problem for the similar conditions as in our previous multi-objective optimization problem [8]. The upper bound on the volume in the reactor is the limiting factor which determines the maximal volume of the feed as well as the terminal times of core and shell formation steps. The core formation step ends when the half of the available feed is consumed. In [8], the best identified feeding profile was applied to the reactor and it was found that the diameters of core and shell of produced polymer particles were $70 \mathrm{~nm}$ and $82 \mathrm{~nm}$, respectively. The restriction of achieving 
Table 2: Scenarios of optimal control problem (12) considered in this study.

\begin{tabular}{cccccc}
\hline & $V_{R, \max }\left[\mathrm{dm}^{3}\right]$ & $d_{p 1}[\mathrm{~nm}]$ & $d_{p 2}[\mathrm{~nm}]$ & $\varepsilon$ & Temperature $\left[{ }^{\circ} \mathrm{C}\right]$ \\
\hline S1 & 0.73 & - & - & 10 & 70 \\
S2 & $\infty$ & 70 & 82 & 10 & 70 \\
S3 & $\infty$ & 70 & 82 & 5 & 70 \\
S4 & $\infty$ & 70 & 82 & 1 & 70 \\
S5 & $\infty$ & 70 & 82 & 10 & 80 \\
\hline
\end{tabular}

the aforementioned core and shell diameters is considered in the scenario S2 while the upper limit on the reactor volume is removed. This is done in order to investigate a case where core and shell diameters are specified. As shown later, simultaneous consideration of both constraints (maximal reactor volume, core/shell diameter) would lead to unsatisfactory tracking profiles of glass transition temperature. For scenarios S2-S5, the available feed volume is considered to be infinite while its composition (expressed in terms of concentrations) is the same as in Table 1. In both scenarios S1 and S2, relaxed constraint on starving conditions period is used and this is set equal to ten. In scenarios S3 and S4, we study the effect of tightening this constraint while $\varepsilon$ takes the values of five (S3) and one (S4). Scenarios S1-S4 consider that the reaction takes place under isothermal conditions of $70^{\circ} \mathrm{C}$. In scenario S5 the effect of an increased reactor temperature to $80^{\circ} \mathrm{C}$ is analyzed.

\subsection{Scenario $S 1$ - Effect of reactor volume constraints}

Figure 2 shows the optimal feeding profile and the corresponding evolution of glass transition temperature, monomers global conversion, and growth 


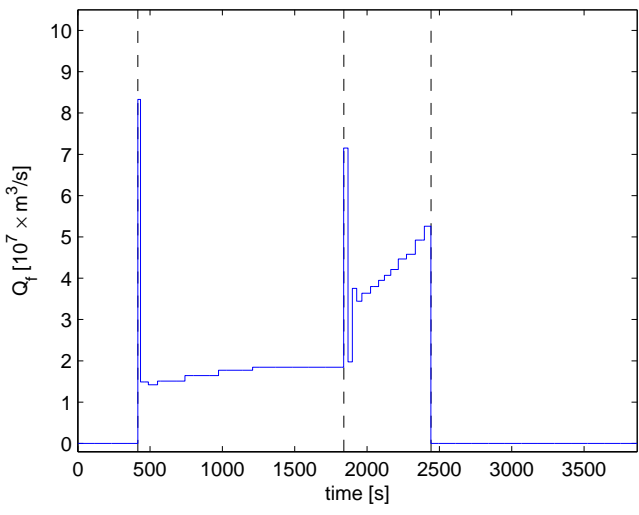

(a) feeding rate

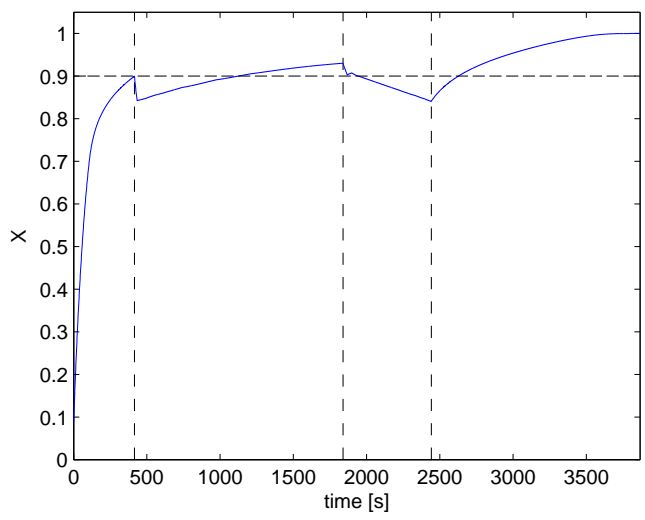

(c) overall conversion of monomers

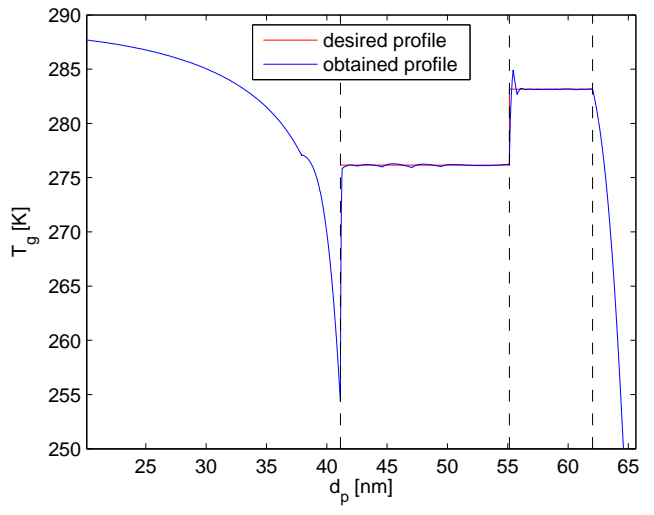

(b) glass transition temperature

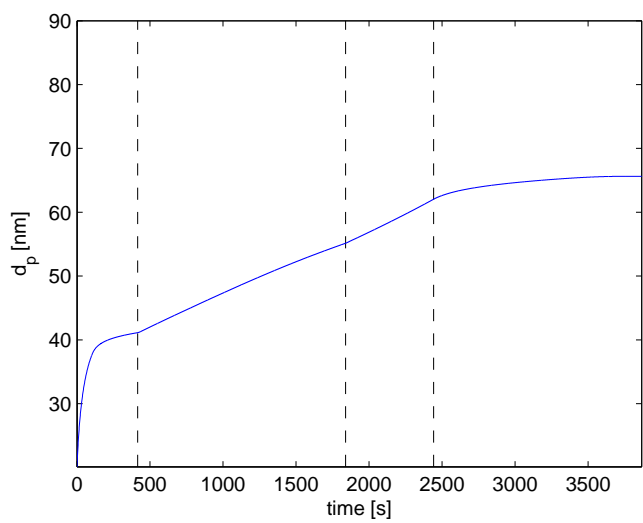

(d) particles diameter

Figure 2: Optimal feeding policies and the corresponding state trajectories for the case of upper limit constraint on reactor volume (scenario S1). 


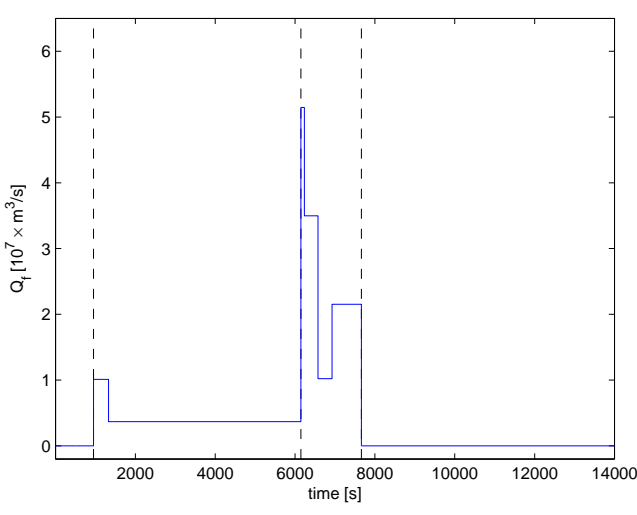

(a) feeding rate

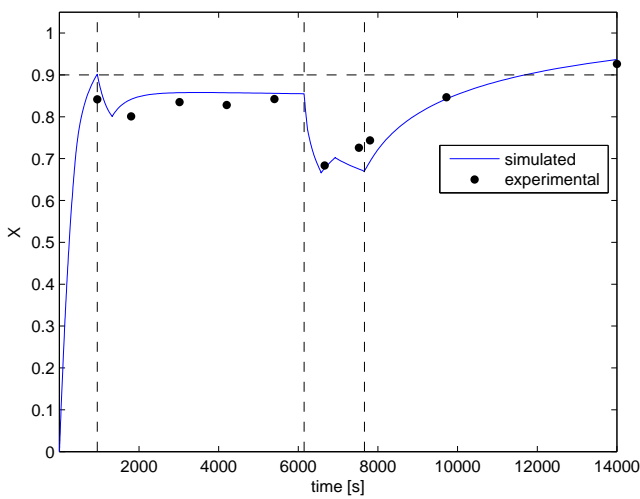

(c) overall conversion of monomers

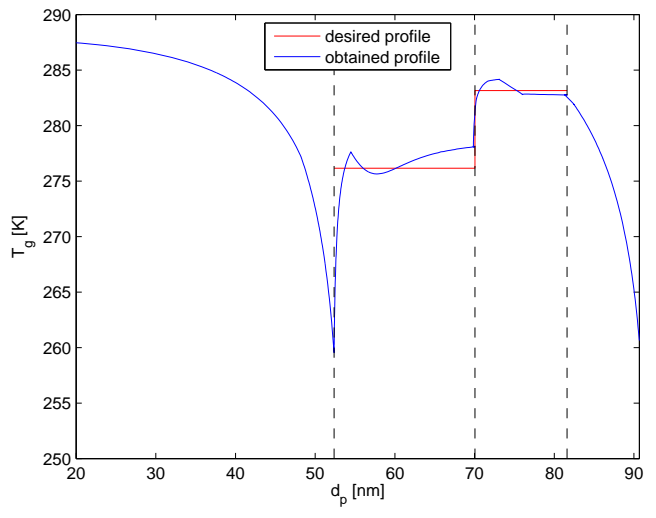

(b) glass transition temperature

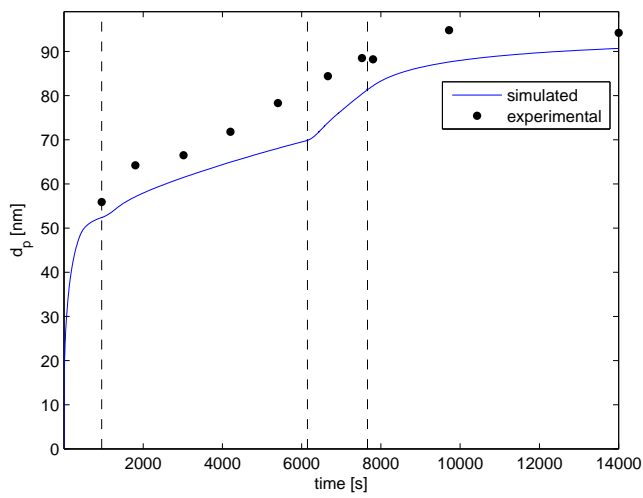

(d) particles diameter

Figure 3: Experimental results for open-loop application of optimal feeding policies taken from $[8]$. 
of particle diameter. The shape of the feeding profile exhibits the typical behavior of the solution of tracking problems of nonlinear systems. In the core formation step, a short impulse of feeding hits the constraint on feed flowrate. Then the feeding rate drops down and it follows quasi-linear trajectory until the end of this stage. To uncover such impulse-type behavior of control, we decreased the time-length restriction of the first control segment in core formation phase to 15 seconds which showed to be short enough. Similar behavior of optimal control profile could be observed for the shell formation step. The minimum length of a control segment is, however, kept equal to 30 seconds which translates to the initial oscillation on first two control segments. This effect is reflected in the tracking of the desired profile of glass transition temperature. This is found to be satisfactory with an overshoot being a direct consequence of control oscillation. Further, we may observe for monomers global conversion rate that even when starving conditions are not strongly enforced in the core formation step, the overall conversion profile evolves in the close neighborhood of $90 \%$.

When these results are compared to those obtained in our previous study [8] (shown in Fig. 3), we may observe a reduction in reaction time and, correspondingly, smaller average diameters of produced particles. These rather big differences are attributed to less fine discretization of the feeding profile in the previous work [8] while the resolution of computationally demanding multi-objective optimal control problem was performed. We present the experimental results obtained by open-loop application of the optimal control profile computed in [8]. Comparison of experimental and simulation results proves that the used model of copolymerization reaction is capable of predic- 
tion of the process states.

\subsection{Scenario S2 - Effect of prescribed core/shell diameters}

Figure 4 shows the optimal feeding profile and the corresponding evolution of glass transition temperature, monomers global conversion, and growth of particle diameter. The shape of optimal feeding profile shows similar behavior to the previous case with a difference in the tracking of the desired glass transition temperature profile in the core formation step. Restrictions to time-length of PWC segments are kept at thirty seconds. This behavior is attributed to the removed constraint on feed consumption and to the introduction of the constraints on a particle diameter which accordingly prolongates the step duration (from one thousand to four thousand seconds). It can be seen that all the profiles in Figs. 2 and 4 are identical until one thousand seconds of reaction time. We can observe that a satisfactory profile of $T_{g}$ was obtained while respecting the constraints on diameter of core and shell of produced particles. We can also conclude that the plot of monomers global conversion shows that starving conditions are not strictly maintained in the core formation step. This behavior occurs due to a relatively loose relaxation of the corresponding constraints $(\varepsilon=10)$.

\subsection{Scenarios $S 3 \& S_{4}$ - Effect of enforced starving conditions}

Figure 5 shows a comparison of optimal feeding profiles and their corresponding evolutions of glass transition temperature, monomers global conversion, and growth of particle diameter for the cases when starving conditions are enforced by tightening the relaxation with parameter $\varepsilon$ being respectively equal to five and one. The shape of optimal feeding profile and its structure 


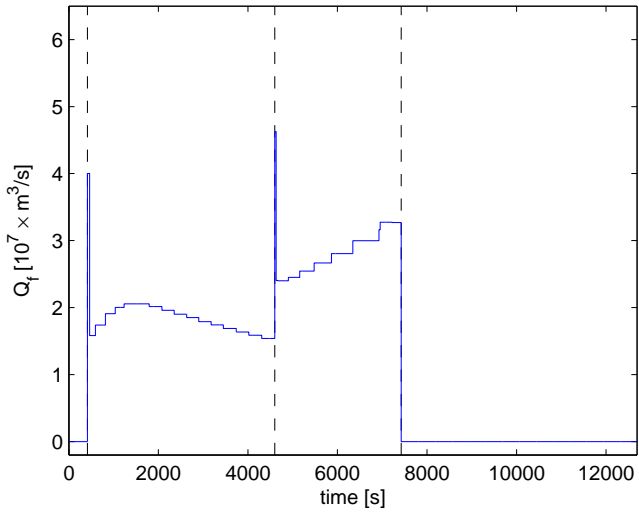

(a) feeding rate

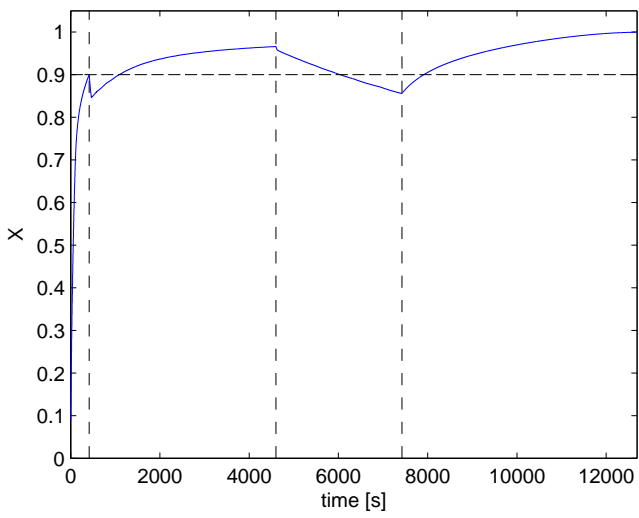

(c) overall conversion of monomers

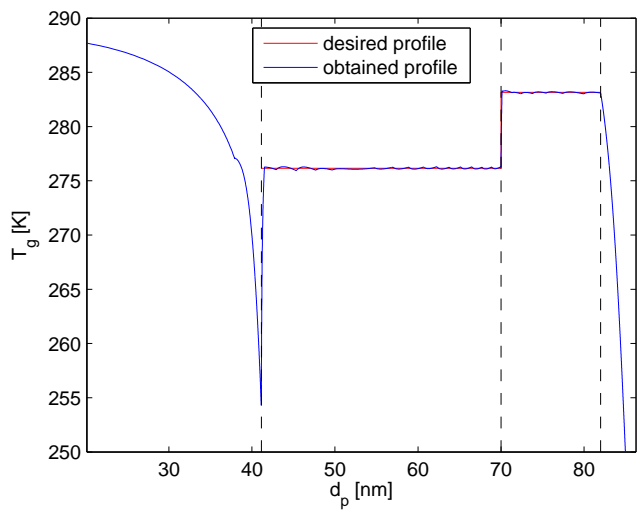

(b) glass transition temperature

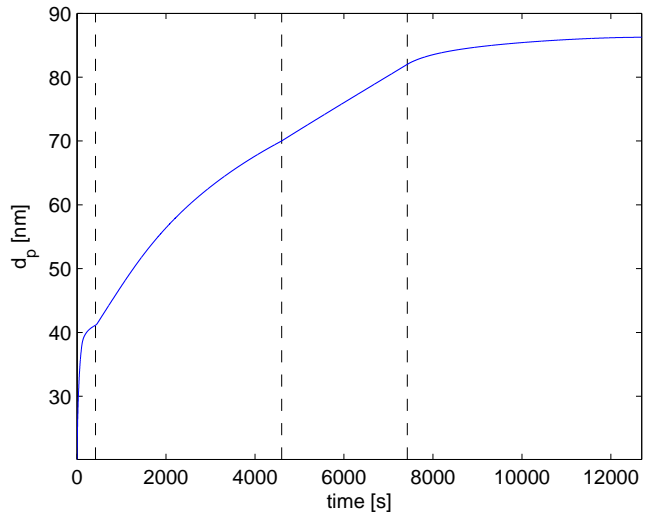

(d) particles diameter

Figure 4: Optimal feeding policy and the corresponding state trajectories for scenario S2 $(\varepsilon=10)$. 

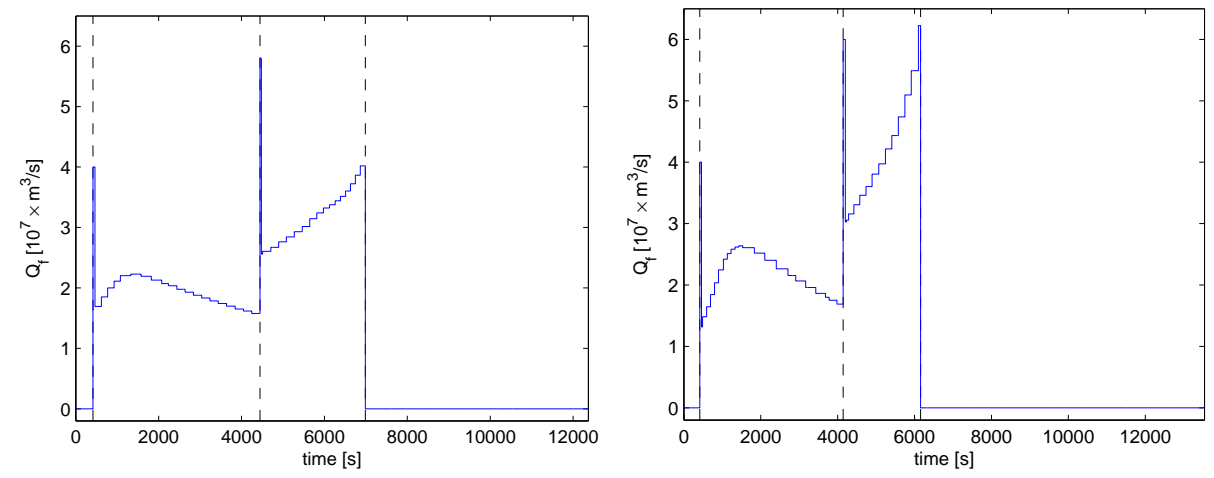

(a) feeding rate
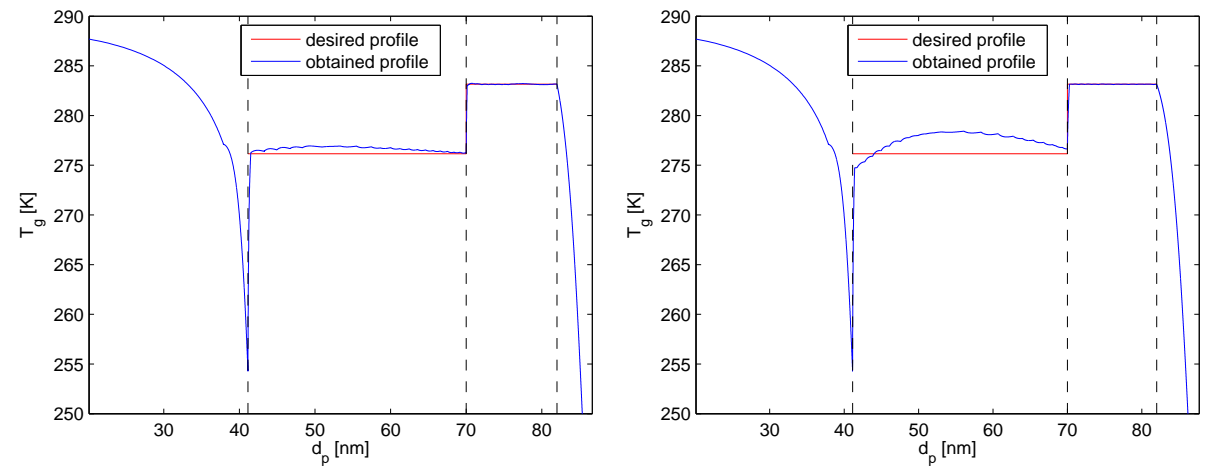

(b) glass transition temperature
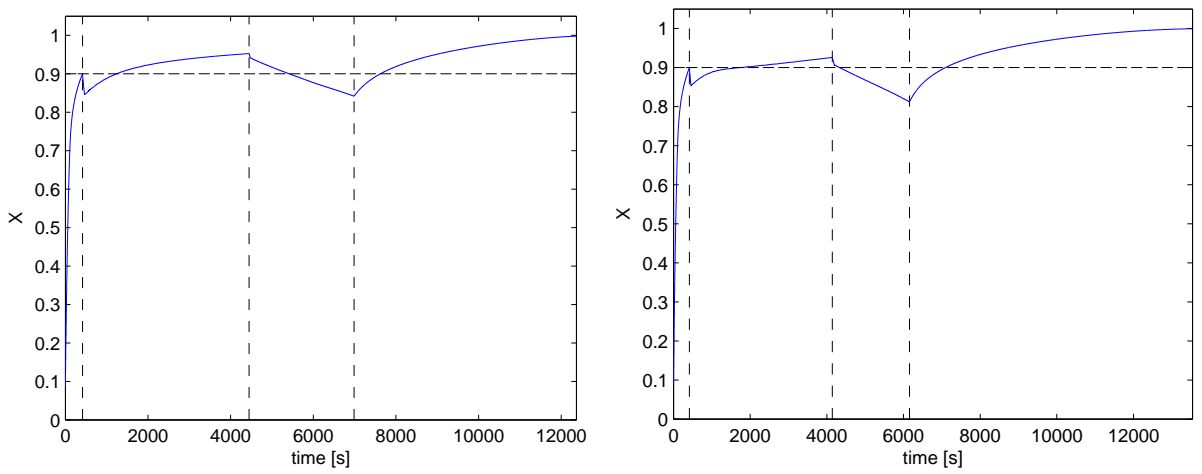

(c) overall conversion of monomers

Figure 5: Comparison of optimal feeding policies and the corresponding state trajectories for scenarios S3 and S4. Left plots: S3, $\varepsilon=5$, right plots: $\mathrm{S} 4 \varepsilon=1$. 
show a big portion of similarity with respect to the optimal feeding profiles observed for previous cases. The first difference is observed, as expected, in the feeding throughout the core formation step where more monomer is fed into the reactor in order to get the starving conditions satisfied close to the end of core formation step. This result was pointed out in [13].

We can very easily see the effect of tightening the constraint relaxation on starving conditions in this step. As it can be predicted, the monomers global conversion evolves closer to the 90\% "setpoint". Furthermore, due to aforementioned of trade-off between obtained quality and quantity of the copolymer, the tracking of glass transition temperature desired profile becomes worse when decreasing $\varepsilon$. We can see that it is then again possible to obtain ideal tracking of $T_{g}$ profile during the shell formation step. This is expected since no other constraints on monomer trajectory evolution are active. An interesting observation here is that the price we have to pay for stronger enforcement of starving conditions in the core formation phase, is the increased amount of feeding during shell formation step. Beside the increased amount of produced polymer, this also leads to increased reaction time which is evident from Figure 5 and stands for more than one thousand seconds difference compared to the previous case. This is an interesting behavior of the solution to optimal control problem (12) which points out the nonlinear nature of the process and deserve a certain level of attention when the production of particles with core-shell morphology is desired.

\subsection{Scenario S5-Effect of reaction temperature}

The minimization (or reduction) of reaction time for chemical processes is traditionally achieved by varying the operating conditions like reaction 


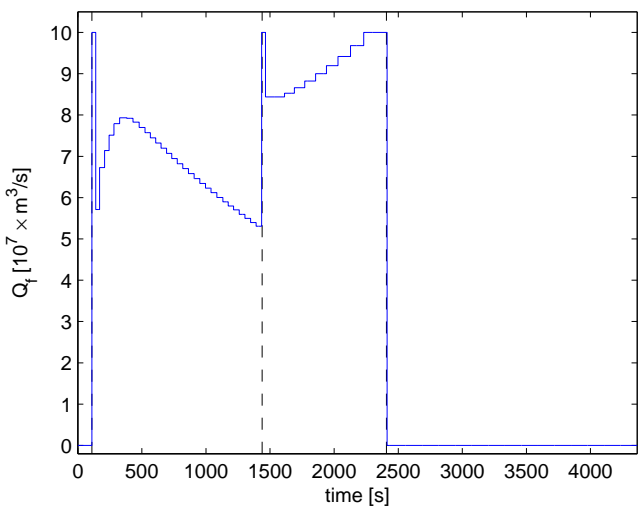

(a) feeding rate

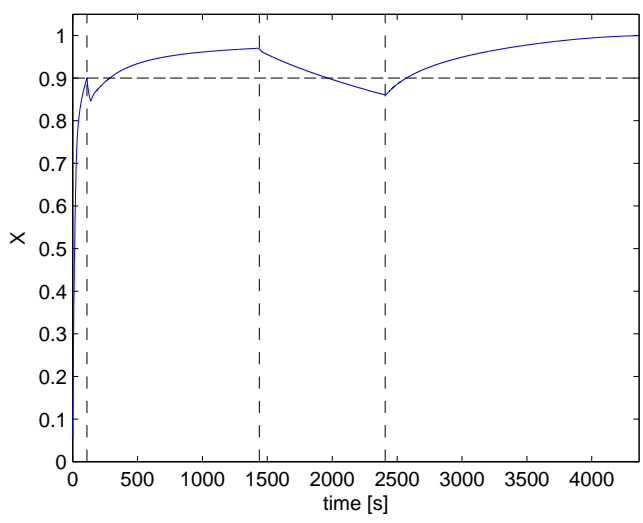

(c) overall conversion of monomers

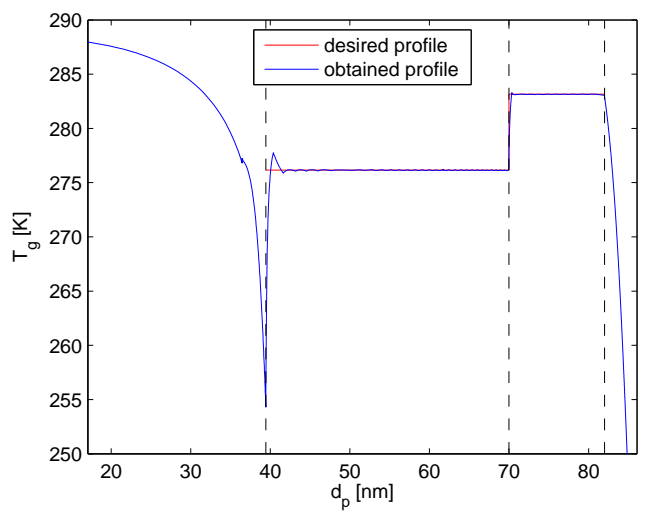

(b) glass transition temperature

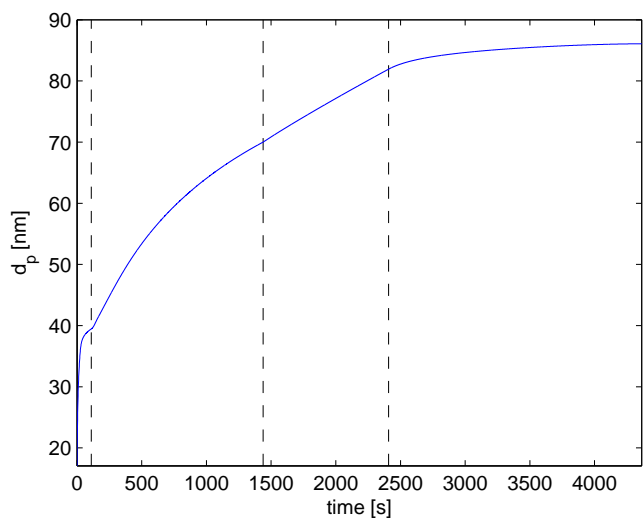

(d) particles diameter

Figure 6: Optimal feeding policy and the corresponding state trajectories for scenario S5 (reaction at $\mathrm{T}=80^{\circ} \mathrm{C}$ ). 
temperature or pressure. It is the aim of the following analysis to investigate the effect of increased temperature for the studied emulsion copolymerization reaction and to prove the ability of control to maintain the previously observed achievement of product-quality goals. The reaction takes place at $80^{\circ} \mathrm{C}$ instead of $70^{\circ} \mathrm{C}$ which was used in the previous problems.

Figure 6 shows the optimal feeding profiles and the corresponding evolution of glass transition temperature, monomers global conversion, and growth of particle diameter where the reactor is assumed to be kept isothermal at $80^{\circ} \mathrm{C}$. As it can be seen, the same shape and structure of the optimal feeding profile of monomers are present as it was the case for the reaction at $70^{\circ} \mathrm{C}$. As expected, the reaction time is significantly reduced. Moreover, a very good tracking of the desired $T_{g}$ profile can be achieved. This needs, however, to broaden the limits of the system since higher feeding rates are expected due to increased reaction rates. For the purpose of this analysis, we increased the maximal feeding rate to $Q_{f, \max }=10 \times 10^{-7} \mathrm{~m}^{3} \mathrm{~s}^{-1}$. We can observe practically negligible oscillation of obtained $T_{g}$ profile in the beginning of core formation step which occurs due to the limits on time-length of PWC control segment of discretized control profile.

The fact that we achieved similar control profiles in all considered operational scenarios shows very interesting property of the solution to problem (12). It also provides a vision for exploiting this behavior in order to identify a closed-loop control policy based on the identification of active constraints and sensitivity-seeking control arcs. A closed-loop application of such control will be desired to act against the effect of uncertainties which might occur. A possible approach to this problem was recently shown in [23]. 


\section{Conclusions}

In this work, an analysis was performed on optimal operation of fed-batch emulsion copolymerization reactor of styrene and butyl acrylate in the presence of chain transfer agent. We studied several scenarios of operational constraints and requirements. The aim was to derive an optimal control (feeding) profile of the considered copolymerization reactor for tracking of the desired profile of glass transition temperature while satisfying given constraints and maximizing the final overall conversion of the monomers. The second aim was to observe the behavior of the optimal feeding profile under several operational scenarios. Dynamic optimization was performed via control vector parameterization method implemented within gPROMS environment in order to solve the arising optimal control problem. It was possible to achieve good convergence of numerical NLP solver mainly due to the builtin evaluation of sensitivity equations that provided gradient information of solved optimal control problems. The experimental results were presented in order to evidence the accuracy of predictions provided by the employed process model. This evidence is particularly strong when we take into account the fact that the presented experimental results are obtained while applying optimal open-loop control profiles (optimized feeding rates) to the real plant.

As mentioned, the effect of different aspects of operational constraints and demands was studied. It was shown that the particles with desired core-shell morphology can be produced in several process setups and that the feeding of monomers can be adjusted to account for various operational requirements. Moreover, it was observed that the solution to the optimal control problem behaves similarly in all treated scenarios of different operational conditions 
and requirements. This reveals a possibility of designing a tailored feedback policy for control of this process. Such policy would require to identify the set of active constraints and process modes (because of the switching behavior of the process). It is then a part of our future work to derive such nonlinear control laws and to test them on the real plant.

\section{List of Symbols}

a Fox and Flory adjustment parameter

$C T A_{p} \quad$ molecule of CTA in the particles

$C T A_{p}^{\bullet} \quad$ radical of CTA in the particles

$[C T A]_{f}$ concentration of the transfer agent in the feed

$d_{p} \quad$ average particles diameter

$f_{a q i} \quad$ the molar fraction of $i$ th monomer in the aqueous phase

$I \quad$ total number of moles of initiator in the aqueous phase

$[I]_{f} \quad$ concentration of the initiator in the feed

$k_{c p} \quad$ capture rate coefficient of free radicals from the $i$ th monomer by particles

$k_{C T A p} \quad$ rate coefficient of transfer to CTA

$k_{d} \quad$ initiator decomposition rate constant

$k_{\text {desi }}$ desorption rate coefficient of the radical ended by the $i$ th monomer

$k_{i} \quad$ transfer coefficient of free radicals formed by one monomer unit

$k_{N} \quad$ nucleation rate coefficient

$k_{p i j} \quad$ propagation rate coefficient of the $j$ th monomer with free radical ended by $i$ th monomer 
$k_{t c i j} \quad$ rate coefficient of termination by combination

$k_{t d i j} \quad$ rate coefficient of termination by disproportionation

$k_{t i j} \quad$ termination rate coefficient

$k_{t r m i j}$ transfer to monomer rate coefficient (radicals ended by the $i$ th monomer to the $j$ th monomer)

$k_{Z a q} \quad$ inhibition rate coefficient in aqueous phase

$k_{Z p} \quad$ inhibition rate coefficient in organic phase

$L_{k} \quad k$ th normalized moment of the macromolecules

$\bar{M}_{n} \quad$ number average molecular weight

$\bar{M}_{p} \quad$ weight average molecular weight

$M_{i} \quad$ total number of moles of the $i$ th monomer in the reactor

$M_{T i} \quad$ total number of moles of the $i$ th monomer fed to the reactor

$M_{M}^{i} \quad$ molecular weight of the $i$ th monomer

$\left[M_{i}\right]_{f} \quad$ concentration of the $i$ th monomer in the feed

$N_{p} \quad$ total number of moles of particles

$N_{m} \quad$ total number of macromolecules

$\bar{n} \quad$ average number of free radicals in a particle

$\tilde{n} \quad$ average number of pairs of free radicals in a particle

$P \quad$ molecule of polymer

$Q_{f} \quad$ total feed rate of monomers, inhibitor, transfer agent, and surfactant

$Q_{f I} \quad$ feed rate of initiator

$R^{\bullet} \quad$ free radical in organic phase

$R_{a q} \quad$ number of moles of free radicals in the aqueous phase

$R_{a q}^{\bullet} \quad$ free radical in aqueous phase 
$\mathcal{R}_{d} \quad$ thermal decomposition rate of initiator in the aqueous phase

$\mathcal{R}_{N} \quad$ total micellar nucleation rate

$R_{p i} \quad$ the number of moles of free radicals of $i$ th monomer in the particles

$\mathcal{R}_{p} \quad$ total propagation rate

$\mathcal{R}_{d} \quad$ thermal decomposition rate of initiator in the aqueous phase

$\mathcal{R}_{C T A p}$ transfer agent consumption rate in particles

$\mathcal{R}_{t} \quad$ total termination rate

$\mathcal{R}_{T C} \quad$ total termination rate by combination

$\mathcal{R}_{T D} \quad$ total termination rate by disproportionation

$\mathcal{R}_{\text {trm }} \quad$ total transfer to monomer rate

$\mathcal{R}_{Z a q} \quad$ inhibition consumption decomposition of initiator in the aqueous phase

$\mathcal{R}_{Z p} \quad$ inhibitor consumption rate in particles

$S$ total number of moles of the surfactant in the reactor

$[S]_{f} \quad$ concentration of the surfactant in the feed

$T$ temperature of the reaction mixture

$T_{g} \quad$ glass transition temperature

$V_{R} \quad$ total volume engaged in the reactor

$W_{i} \quad$ mass fraction of the $i$ th monomer in the copolymer

$X \quad$ overall mass conversion

$Z_{a q} \quad$ molecule of inhibitor in aqueous phase

$Z_{p} \quad$ molecule of inhibitor in organic phase

$Z_{p}^{\bullet} \quad$ radical of inhibitor in organic phase

$[Z]_{f} \quad$ concentration of the inhibitor in the feed 


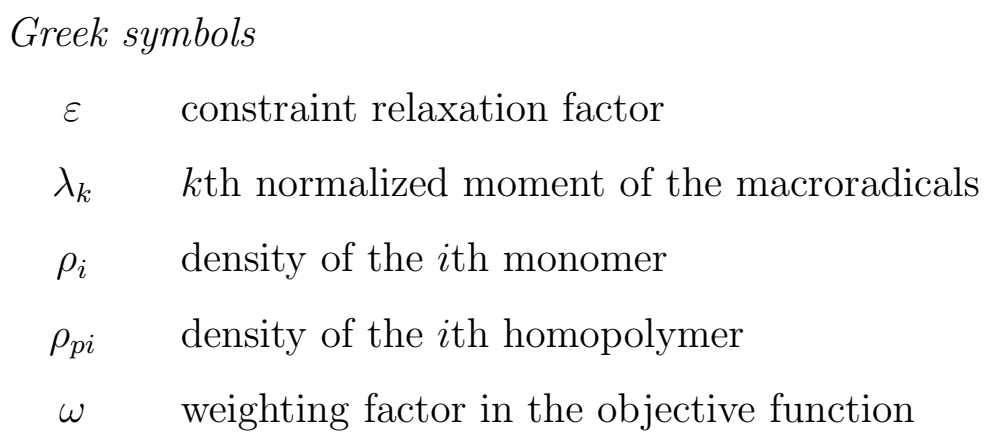

\section{Subscripts}

$f \quad$ feed

f final (time, state)

\section{Abbreviations}

CTA chain transfer agent

CVP control vector parameterization

DAE differential-algebraic equations

MWD molecular weight distribution

NLP nonlinear programming

PSD particle size distribution

PWC piece-wise constant

\section{Acknowledgments}

The authors gratefully acknowledge the contribution of the Scientific Grant Agency of the Slovak Republic under the grant 1/0053/13 and the Slovak Research and Development Agency under the projects APVV-0551- 
11 and SK-FR-0004-11.

[1] S. C. Thickett, R. G. Gilbert, Emulsion polymerization: State of the art in kinetics and mechanisms, Polymer 48 (24) (2007) 6965-6991. doi:10.1016/j.polymer.2007.09.031.

[2] W. D. Harkins, A general theory of the mechanism of emulsion polymerization1, Journal of the American Chemical Society 69 (6) (1947) 1428-1444. doi:10.1021/ja01198a053.

[3] M. A. Dubé, J. B. P. Soares, A. Penlidis, A. E. Hamielec, Mathematical modeling of multicomponent chain-growth polymerizations in batch, semibatch, and continuous reactors: A review, Industrial \& Engineering Chemistry Research 36 (4) (1997) 966-1015. doi:10.1021/ie960481o.

[4] J. M. Asua, Emulsion polymerization: From fundamental mechanisms to process developments, Journal of Polymer Science Part A: Polymer Chemistry 42 (5) (2004) 1025-1041. doi:10.1002/pola.11096.

[5] J. Gao, A. Penlidis, Mathematical modeling and computer simulator/database for emulsion polymerizations, Prog. Polym. Sci 27 (2002) 403-535. doi:10.1016/S0079-6700(01)00044-2.

[6] C. S. Chern, Emulsion polymerization mechanisms and kinetics, Progress in Polymer Science 31 (5) (2006) 443-486. doi:10.1016/j.progpolymsci.2006.02.001.

[7] B. Benyahia, M. A. Latifi, C. Fonteix, F. Pla, S. Nacef, Emulsion copolymerization of styrene and butyl acrylate in the presence of a chain 
transfer agent. part 1: Modelling and experimentation of batch and fedbatch processes, Chemical Engineering Science 65 (2) (2010) 850-869. doi:10.1016/j.ces.2009.09.036.

[8] B. Benyahia, M. A. Latifi, C. Fonteix, F. Pla, Multicriteria dynamic optimization of an emulsion copolymerization reactor, Computers \& Chemical Engineering 35 (12) (2011) 2886 - 2895. doi:10.1016/j.compchemeng.2011.05.014.

[9] J. H. Park, K. H. Oh, S. H. Kim, A. Cyriac, J. K. Varghese, M. W. Hwang, B. Y. Lee, Morphology control of polymer particles in ethylene/carbon monoxide copolymerization, Angewandte Chemie International Edition 50 (46) (2011) 10932-10935. doi:10.1002/anie.201105270.

[10] D. C. Sundberg, Y. G. Durant, Thermodynamic and kinetic aspects for particle morphology control, in: J. M. Asua (Ed.), Polymeric Dispersions: Principles and Applications, Vol. 335 of NATO ASI Series, Springer Netherlands, 1997, pp. 177-188. doi:10.1007/978-94-011-5512$0 \_12$.

[11] M. Okubo, Control of particle morphology in emulsion polymerization, Makromolekulare Chemie. Macromolecular Symposia 35-36 (1) (1990) 307-325. doi:10.1002/masy.19900350119.

[12] X.-D. He, X.-W. Ge, M.-Z. Wang, Z.-C. Zhang, Morphology control of hollow polymer latex particle preparation, Journal of Applied Polymer Science 98 (2) (2005) 860-863. doi:10.1002/app.22181. 
[13] R. Paulen, B. Benyahia, M. A. Latifi, M. Fikar, Dynamic optimization of semi-batch emulsion copolymerization reactor for styrene/butyl acrylate in the presence of a chain transfer agent, in: A. Kraslawski, I. Turunen (Eds.), 23rd European Symposium on Computer Aided Process Engineering, Elsevier, Radarweg 29, PO Box 211, 1000 AE Amsterdam, The Netherlands, Vol. 32, 2013, pp. 721-726.

[14] T. G. Fox, P. J. Flory, Second-order transition temperatures and related properties of polystyrene, Journal of Applied Physics 21 (1950) 581-591. doi:10.1063/1.1699711.

[15] C. J. Goh, K. L. Teo, Control parameterization: A unified approach to optimal control problems with general constraints, Automatica 24 (1) (1988) 3-18. doi:10.1016/0005-1098(88)90003-9.

[16] Process Systems Enterprise, gPROMS, www.psenterprise.com/gproms (1997-2009).

[17] B. Houska, H. Ferreau, M. Diehl, ACADO Toolkit - An Open Source Framework for Automatic Control and Dynamic Optimization, Optimal Control Applications and Methods 32 (3) (2011) 298-312.

[18] P. E. Rutquist, M. M. Edvall, PROPT - Matlab Optimal Control Software, tomdyn.com (2010).

[19] M. Diehl, D. B. Leineweber, A. Schäfer, MUSCOD-II Users' Manual, Universität Heidelberg (2001).

[20] M. Fikar, M. A. Latifi, Users guide for fortran dynamic optimisation 
code dyno, Technical report mf0201, LSGC CNRS, Nancy (France), STU Bratislava (Slovakia) (2002).

[21] M. Čižniar, M. Fikar, M. A. Latifi, MATLAB Dynamic Optimisation Code DYNOPT. User's Guide, KIRP FCHPT STU, STU in Bratislava (2006).

[22] P. I. Barton, C. K. Lee, Modeling, simulation, sensitivity analysis and optimization of hybrid systems, ACM Transactions on Modeling and Computer Simulation 12 (2001) 256-289. doi:10.1145/643120.643122.

[23] S. Deshpande, D. Bonvin, B. Chachuat, Directional input adaptation in parametric optimal control problems, SIAM Journal on Control and Optimization 50 (4) (2012) 1995-2024. doi:10.1137/110820646. 